\title{
Primary Fibrosarcoma of the Testis and Other Scrotal Contents: \\ A Review and Update
}

\author{
Anthony Kodzo-Grey Venyo \\ North Manchester General Hospital, Department of Urology, Manchester, M8 5RB, United \\ Kingdom.
}

Email: akodzogrey@yahoo.co.uk

\section{ABSTRACT}

Primary fibrosarcoma of the scrotal contents has been sporadically reported with short-term and medium-term follow-up data. Generally, the tumours had presented insidiously as slow-growing intra-scrotal masses and some of them had presented as a recent increase in the size of a longstanding scrotal masses. The results of serum beta-human chorionic gonadotrophin, alphafetoprotein, and lactate dehydrogenase tend to be normal. Radiology imaging of the scrotal masses tended to show a heterogeneous mass within the testis or localized a specific site in the scrotum displacing the ipsilateral testis to the contralateral side. The tumours have generally been treated by means of radical orchidectomy (radical excision of tumour with tumour-free surgical excision margin) alone but some individuals have undergone adjuvant radiotherapy alone plus or minus adjuvant chemotherapy. Diagnosis is based upon the histopathology and the immunohistochemistry examination features of the tumour. The long-term survival rates of patients who have been treated for fibrosarcoma tend to be low despite radical excision, radiotherapy, and chemotherapy. Tumours that are large-sized, poorly differentiated as well as tumours that have high mitotic figures tend to have an inferior prognosis. Radiotherapy-induced fibrosarcoma of the inguinoscrotal content has been reported pursuant to radical orchidectomy plus adjuvant radiotherapy for seminoma of the testis which would indicate that perhaps radiotherapy should not be used. There is a need to identify though a meticulous research specific combination chemotherapy medicaments that would effectively destroy fibrosarcoma tumour cells at the initial stage of treatment. The possibility of the use of immunotherapy as additional treatment should be investigated through a multi-centre global study so that patients, who have intra-scrotal fibrosarcomas that are large-sized, poorly differentiated and that contain high mitotic activity could be treated with neoadjuvant chemotherapy followed by radical orchidectomy and adjuvant chemotherapy plus or minus immunotherapy in order to improve the long-term survival.

Key Words: Primary; Fibrosarcoma; Testis; Tunica; Spermatic cord; Radical orchidectomy; Adjuvant Chemotherapy; Ultrasound scan.

\section{Introduction}

Fibrosarcoma which is also referred to as fibroblatic sarcoma is a terminology that is utilized for a malignant mesenchymal tumour which is derived from fibrous connective

tissue and which is typified by the presence of immature proliferating fibroblasts or undifferentiated anaplastic spindled cells in a storiform format. Fibrosarcoma can develop 
as a soft-tissue growth or as a primary or metastatic bone tumour. [1] It has been iterated that previously fibrosarcoma used to be diagnosed more often than it is diagnosed these days which presumably is because fibrosarcoma can be more confidently differentiated by the histopathology examination features of the tumour from lesions that simulate fibrosarcoma for examples desmoid tumours, malignant fibrous histiocytoma, [2] malignant schwannoma, and high-grade osteosarcoma. [1] The commonest treatment option that has been adopted for fibrosarcoma has remained complete radical excision of the tumour ensuring there is clear or tumour free surgical resection margin. It has been stated that the long-term survival and the functional outcome ensuing treatment for the treatment of fibrosarcoma do depend upon various interrelated factors. [1] [3] [4] Some of these stipulated factors include the size of the tumour, the location of the tumour, the histopathology grading of the tumour, presence or absence of metastases for example lung metastases. The aforementioned factors tend to be considered with regard to the careful stating and evaluation of the tumour and these factors do determine the success or failure of the treatment with regard to the achieving of good local control of the disease and prevention of subsequent development and spread of metastatic disease. [1]. It has been iterated that continued advances which are being made with regard to the molecular biology of sarcomas could further explain the clinical and biological behaviour of the various sub-types of fibrosarcoma and would eventually establish better solutions to the treatment of the respective sub-types of fibrosarcoma. [1]Soft tissue fibrosarcoma is stated to be associated with a $40 \%$ to $60 \% 5$ year survival rate among adults [1] It has also been stated that the 5-year survival for infantile fibrosarcoma of soft-tissues is greater than $80 \%$ [1] It has been documented that if all grades of fibrosarcoma are taken into consideration, primary fibrosarcoma of the bone tends to be associated with inferior prognosis in comparison with osteosarcoma of with a 5-year survival of $65 \%$. [1] It has additionally been iterated that with regard to high-grade primary fibrosarcoma, the 10-year survival rate tends to be less than $30 \%$ and that secondary fibrosarcoma tends to be associated with a very poor prognosis as well as the 10 -year survival rate is less than $10 \%$. [1] It has furthermore been stated that with regard to congenital fibrosarcoma of bone in children, the outcome which tends to be linked to the age of the patient and the time to the diagnosis of the disease tends to be much better, with the disease being associated with long-term survival rates higher than $10 \%$. [1]Adult-type fibrosarcoma tends to be an uncommonly encountered as well as a highly aggressive subtype of soft tissue sarcomas and diagnosis of the disease always tends to be established by the exclusion of other malignancies. [5] It has been documented that the likelihood of misdiagnosis between tumour entities that simulate fibrosarcoma is high and on many occasions tend to lead to inappropriate treatment of the tumour. [5] With regard the features of fibrosarcoma, it has been stated that when fibrosarcoma is diagnosed appropriately, the overall outcome of the patient pursuant to treatment tends to be generally poor as well as that fibrosarcoma is typified by its low sensitivity to radiotherapy and chemotherapy as well as by its high recurrence rate. For the aforementioned reasons, it has been recommended that new methods of treatment should be identified to improve upon the outcome of the disease. [5]

Considering that primary fibrosarcoma of the scrotal contents is extremely rare majority of 
clinicians would be unfamiliar with the disease and its biological behaviour and they would also tend not to consider of having a high index of suspicion for primary fibrosarcoma as being a possible cause of an intra-scrotal mass which could easily lead to misdiagnosis of the disease. Considering the rarity of primary fibrosarcoma of the scrotal content organs, it would appear as if there is no universal global consensus opinion regarding the best treatment options for the malignancy.

The ensuing article on primary fibrosarcoma of the intra-scrotal organs is divided into two parts (A) Overview which has discussed various general aspects of fibrosarcoma and (B) which contains miscellaneous narrations and discussions from some case reports, case series and studies related to primary fibrosarcoma of the scrotal contents.

\section{Aim}

To review and update the literature on fibrosarcoma that has involved the intrascrotal contents including the testis, tunica, epididymis, spermatic cord, and any other intra-scrotal organ or structure.

\section{Methods}

Various internet databases were searched including Google; Google Scholar; yahoo; and PUBMED. The search words that were used included: Fibrosarcoma; Fibrosarcoma of the testis; Fibrosarcoma of tunica; fibrosarcoma of epididymis, fibrosarcoma of the spermatic cord; para-testiculafibrosarcoma; intrascrotal fibrosarcoma. Seventeen references were identified which were used to write a review and update of the literature that has been divided into two parts (A) Overview which has discussed various general aspects of fibrosarcoma and (B) Miscellaneous narrations and discussions from some case reports, case series, and studies related to primary fibrosarcomas that had affected the scrotal contents.

\section{Results / Review and Update of} Literature

\section{(A) Overview}

General comments / Definition

- Fibrosarcoma is a terminology that is used for a malignant tumour of fibroblasts that have herringbone architecture and variable amounts of collagen [6]

- It has been iterated that fibrosarcomas are uncommon and they to account for up to $3 \%$ of sarcomas in adults [6] pathology outlines.com]

- It has been documented that some people tend to limit the diagnosis of fibrosarcoma to individuals who are older than 10 years but a majority of patients who are diagnosed as having fibrosarcoma tend to be aged between 40 years and 55 years. [6]

- It has been stated that many cases that were previously referred to as fibrosarcoma are actually now regarded as; dedifferentiated liposarcoma, fibromatosis, fibrosarcomatous DFSP, lowgrade fibro-myxoid sarcoma, MPNST, synovial sarcoma, or MFH-pleomorphic [6]

- It has been documented that fibrosarcomas usually tend to affect soft tissues of the lower extremities or trunks of human beings and fibrosarcomas only on rare occasions affect the retroperitoneum or mediastinum organs [6] 
- The finding or report of fibrosarcoma affecting the intrascrotal organs is extremely rare and a majority of clinicians globally would be unfamiliar with the disease and its biological behaviour because they have not encountered the disease occurring within the scrotum.

- It has been stated that $50 \%$ of fibrosarcomas tend to recur and $25 \%$ of fibrosarcomas do metastasize to the lung and bone. [6]

- It has been documented that more metastases of fibrosarcoma do develop if the tumour is histologically more cellular and if the tumour has a higher mitotic activity [6].

\section{Survival}

- It has been stated that generally the 5year survival of fibrosarcomas of soft tissues tends to be about $41 \%$ and the 10 -year survival tends to be about 29\%. [6]

- With regard to survival, it has been iterated that the survival of fibrosarcoma tends to be better if the tumour is superficial and the survival tends to be better if the tumour is upon histopathology examination better differentiated, has low mitotic rate and does not contain any necrosis. [6]

- With regard to primary fibrosarcomas of the intra-scrotal contents, because the tumours are exceedingly rare one cannot make any categorical statement about the prognosis of the tumours but it would be recommended that clinicians should report the cases they encounter with long-term follow-up data to enable a consensus opinion to be made about the biological behaviour of the tumour and its prognosis.

\section{Presentation}

Primary fibromasoma of the scrotal contents may present as follows:

- A testicular mass or lump that may be:

- Painless or previously painless but recently associated with discomfort.

- An intra-scrotal mass or lump that has been growing larger slowly.

- An intra-scrotal lump or mass that which had recently increased in size.

- It is very rare however if there is evidence of inguinal lymph node involvement there may be a history of swelling in the inguinal region.

- The patient may be well otherwise but with the case of intra-scrotal fibrosarcoma associated with metastasis there may be history symptoms related to the site of metastasis but this would generally be rare.

- With regard to cases of radiotherapyinduced fibrosarcoma of an inguinoscrotal content, there be a previous history of radiotherapy to the scrotal area for example for a previously treated seminoma of the testis.

\section{Clinical examination findings:}

- Most cases of primary fibrosarcoma of scrotal contents would tend to be localised disease and in such cases, the general examination and 
systematic examination of the patients would be normal.

- With regard to primary fibrosarcoma of the testis that is localised to the testis, scrotal examination would tend to reveal a hard or firm lump or mass involving part of the involved testis which would be non-tender or occasionally uncomfortable to be palpated and it would tend to be separate from the epididymis and the spermatic cord and upon transillumination the mass would not trans-illuminate but if there is an associated hydrocele there could be evidence of trans-illumination which could suggest presence of a secondary hydrocele or a contemporaneous hydrocele but these clinical findings would be non-specific to diagnose fibrosarcoma of the testis.

- With regard to fibrosarcomaof the tunica, clinical examination of the scrotum would tend to show a lump or mass that feels hard or firm on the surface of the affected part of the testis and displacing the normal feeling testis to the side contralateral to the lump and if the tumour has not become invasive the epididymis and the spermatic cord could be palpated separately and if there is an associated hydrocele or haematocele due to recent clinical examination could reveal evidence of fluid surrounding the testis which would trans-illuminate in cases of associated hydrocele.

- With regard to fibrosarcoma of the epididymis, a scrotal examination would tend to reveal a lump/mass that feels hard or firm and irregular localised within the epididymal region and juxta-posed to the testis and if the tumour is localised it should be possible to palpate the spermatic cord above the lump/mass.

- With regard to fibrosarcoma of the spermatic cord clinical examination would reveal a firm to hard irregular mass located within the spermatic cord and if the tumour is not extremely large the spermatic cord higher than the tumour can be palpated but if the tumour is large and extensive it may simulate an inguinoscrotal hernia and in such situations the spermatic cord would not be palpable above the tumour; nevertheless, it may be possible to palpate the seminal vesicle and testis separately even though the testis could be displaced to the contralateral side.

- With regard to fibrosarcoma of the spermatic cord which has redeveloped many years pursuant to adjuvant radiotherapy following radical orchidectomy for seminoma of the testis, there would be a nonspecific firm or hard mass within the inguinal region which cannot be differentiated easily from an inguinal hernia.

\section{Laboratory Investigations: Urine}

- Urinalysis, urine microscopy, and culture would tend to normal in cases of primary fibrosarcoma of the scrotal contents but if these examinations show evidence of urinary tract infection, the 
infection would be treated to improve the general condition of the patient.

\section{Hematology}

- Full blood count and coagulation screen are routine tests that are undertaken in the general assessment of patients who have primary fibrosarcoma of the scrotal contents and generally the results would be normal but if the results are abnormal this would be investigated appropriately to provide the necessary treatment that would improve the general condition of the patient.

- Full blood count and coagulation screen tend to be undertaken before, during, and after chemotherapy in all patients who undergo chemotherapy for primary fibrosarcoma of the scrotal contents as part of the assessment of the patients.

\section{Biochemistry}

- Serum urea and electrolytes, estimated glomerular filtration rate, liver function tests, and blood glucose are routine tests that tend to be undertaken in the full assessment of all patients who have fibrosarcoma of the scrotal contents and if there is any abnormality it would be adequately investigated and treated accordingly to improve upon the general condition of the patients but on the whole the results would tend to be normal. In situations of advanced disease in which there are extensive retroperitoneal lymph node enlargement and obstruction of the ureter, there could be an impairment of renal function. When there is impairment of renal function due to obstruction of the ureter, radiology imaging would show hydronephrosis and hydroureter and in such situations, percutaneous nephrostomy insertion could be undertaken to improve the renal function and the general condition of the patient as preparation towards the full treatment of the patient.

- Serum Beta Human chorionic Gonadotrophin, Alpha-Fetoprotein, and Lactate dehydrogenase biochemistry tests form part of the initial assessment of patients who have primary fibrosarcoma of the scrotal contents and with regard to pure fibrosarcomas the results would tend to be normal but if there is a cotemporaneous germ cell testicular tumour then one, two or all three tumour marker levels may be raised.

- Serum urea and electrolytes, estimated glomerular filtration rate, liver function tests, and blood glucose are general tests that are undertaken before, during, and after chemotherapy of patients who undergo adjuvant therapy for the disease.

\section{Radiology Chest x-ray}

- Chest X-ray tends to be undertaken as baseline assessment of patients who undergo treatment for intra-scrotal content fibrosarcoma to show there is no metastasis to the lungs especially in developing countries where computed tomography (CT) scan and 
magnetic resonance imaging (MRI) scan is not available or these have broken down and have not yet been repaired. Additionally, chest x-ray and an ultrasound scan of abdomen and pelvis can be undertaken in the follow-up assessment of patients who are undergoing follow-up pursuant to their treatment of curative intent to assess whether or not the patients have developed metastatic disease.

\section{Ultrasound scan}

- Ultrasound scan of the testis and scrotal contents is a commonly used first-line test to assess the scrotal lesion which would tend to show a heterogeneous mass in the testis, tunica, spermatic cord region, or epididymis area and it would also show whether or not there is inguinal lymph node enlargement.

- Ultrasound scan of abdomen and pelvis is a useful way of assessing whether or not there are any metastatic lesions within the abdomen and pelvis or the lymph nodes of the groin and ultrasound scan of abdomen, pelvis, and scrotum can also be undertaken at regular intervals in the follow-up of patients who have undergone treatment for primary fibrosarcoma of the intrascrotal contents. The advantage of this investigation is that there is no radiation involved.

- If the urologist wants to establish the diagnosis of the intra-scrotal tumour in order to plan definitive treatment then ultrasound scan-guided biopsy of the intra-scrotal lesion can be undertaken preceding the definitive surgery and when a histopathology examination diagnosis of fibrosarcoma is made the surgeon would take steps to ensure that complete excision of the tumour is undertaken and perhaps some surgeons and oncologists may enter the patients into clinical trials including radical orchidectomy alone, radical orchidectomy and adjuvant chemotherapy, radical orchidectomy plus radical radiotherapy, radical orchidectomy plus adjuvant chemotherapy plus adjuvant radiotherapy, neoadjuvant chemotherapy plus radical orchidectomy plus neo-adjuvant chemotherapy.

\section{Computed Tomography (CT) Scan}

- Contrast Computed Tomography (CT) scan of the thorax, abdomen, and pelvis are routine radiology investigations that are taken to assess the scrotal lesion for its features in the initial assessment of patients who have fibrosarcoma of the scrotal contents and this would tend to show heterogeneous lesion within the scrotum within the testis, epididymis, tunica, or related to the spermatic cord as well as if there is any lesion in the inguinal region. The contrastenhancing intra-scrotal lesion would illustrate the malignant neoplastic nature of the lesion as opposed to non-contrast enhancing lesions that tend not to be malignant. Additionally, the CT scan of thorax, abdomen, and pelvis would be utilised as the staging 
procedure of the tumour to ascertain whether the malignant intra-scrotal tumour is localised or is associated with local or reginal lymph node involvement of distant metastasis as part of the initial staging of the disease.

- CT scan of thorax, abdomen, and pelvis tends to be undertaken as part of the regular follow-up assessments to ascertain if the malignancy has recurred locally or there has been the development of metastasis to plan further investigations and treatment for the patients.

\section{Magnetic Resonance Imaging (MRI)}

\section{Scan}

- Magnetic Resonance Imaging (MRI) scan of the thorax, abdomen, and pelvis are routine radiology investigations that are taken to assess the scrotal lesion for its features in the initial assessment of patients who have fibrosarcoma of the scrotal contents and this would tend to show heterogeneous lesion within the scrotum within the testis, epididymis, tunica, or related to the spermatic cord as well as if there is any lesion in the inguinal region. The contrastenhancing intra-scrotal lesion would illustrate the malignant neoplastic nature of the lesion as opposed to non-contrast enhancing lesions that tend not to be malignant. Additionally, the MRI scan of thorax, abdomen, and pelvis would be utilised as the staging procedure of the tumour to ascertain whether the malignant intra-scrotal tumour is localised or is associated with local or reginal lymph node involvement of distant metastasis as part of the initial staging of the disease.

- MRI scan of thorax, abdomen, and pelvis tends to be undertaken as part of the regular follow-up assessments to ascertain if the malignancy has recurred locally or there has been the development of metastasis to plan further investigations and treatment for the patients.

\section{Positron Emission Tomography / Computed Tomography (PET / CT) Scan}

- Positron Emission Tomography / Computed Tomography (PET/CT) scan can be undertaken in the followup assessment of patients to ascertain if the patients have developed metastatic lesions anywhere in the body; nevertheless, generally CT scan of thorax, abdomen, and pelvis or MRI scan of thorax, abdomen, and pelvis tend to be undertaken more often in view of the fact that contrast CT scan and contrast MRI scans do provide more details about any lesions that may be encountered.

\section{Bone Scan}

- Isotope bone scan can be undertaken in the follow-up assessment of patients who have undergone treatment for primary fibrosarcoma of an intra-scrotal organ to ascertain if they have developed bone metastasis.

\section{Treatment}

- It has been recommended that with regard to fibrosarcomas of soft tissues the treatment tends to entail radical 
excision, and radiotherapy can be or tends to be undertaken if there is a presence of a residual tumour or the is a tumour positive surgical resection margin [6].

- It has been stated that the use of chemotherapy should be a possibility of the fibrosarcoma upon histopathology examination is found to be a high-grade tumour. [6].

- With regard to primary fibrosarcomas of the intra-scrotal contents it would be said that even though only very few cases have been reported treatment of the malignancy should be trans-inguinal radical orchidectomy with high-ligation and transection of the spermatic cord ensuring there is complete excision of the tumour and if there is evidence of tumour positivity at the surgical resection margin further surgical excision should be undertaken to ensure complete excision of tumour without surgical resection positive margin.

- With regard to primary fibrosarcoma of the intra-scrotal contents even though very few cases have been reported and there is no consensus opinion regarding the treatment some people would argue that since the long-term outcome of the disease tends to be less than $50 \%$ survival there is need for utilization of adjuvant chemotherapy alone or adjuvant chemotherapy plus adjuvant radiotherapy or adjuvant radiotherapy alone. Considering the fact that primary fibrosarcoma of the spermatic cord has been reported in the contra-lateral side of a patient who had undergone adjuvant radiotherapy post radical orchidectomy for a seminoma of the testis, it would be argued that radiotherapy-induced primary fibrosarcoma of the contralateral testis or structures within the radiotherapy field could develop therefore a long period of follow-up would be required if adjuvant radiotherapy of the scrotal contents is undertaken.

- It would also be recommended that a multi-center trial for the treatment of primary intra-scrotal content fibrosarcomas would be required to ascertain if the disease should be treated with radical orchidectomy alone, radical orchidectomy with adjuvant chemotherapy alone, radical orchidectomy plus adjuvant radiotherapy alone, or radical orchidectomy plus adjuvant chemotherapy plus adjuvant radiotherapy.

\section{Macroscopic features}

- It has been stated that macroscopy pathology examination of primary fibrosarcomas, tend to show wellcircumscribed but non-encapsulated tumours. [6]

- It has also been stated that gross examination of specimens of fibrosarcomas, tend to show the fleshy, haemorrhagic necrotic whitetan tumour. [6]

- Some intra-scrotal fibrosarcomas may not contain areas of necrosis or haemorrhage. 


\section{Microscopy examination features}

The microscopic examination features of fibrosarcoma have been summarized as follows;

- Microscopic histopathology examination of the tumour tends to show highly cellular proliferation of tumour cells that are arranged in herringbone fashion in that the cells tend to be arranged within columns of short parallel lines with all of the lines in one column that are sloping one way and the lines that are in the adjacent columns tend to slope the other way. [6]

- It has been stated that microscopic examination of fibrosarcoma tumour does show that the tumour cells contain scant cytoplasm, nuclei that are tapered and elongated and which contain granular chromatin as well as variable nucleoli. [6]

- Mitotic activity tends to be seen in fibrosarcoma tumours and quite often there tend to be abnormal forms of mitotic activity. [6]

- There tends to be variable collagen in fibrosarcomas. [6]

- It has been iterated that usually in fibrosarcoma there usually tends to be no giant cells [6]

- Within fibrosarcomas, there tends not to be evidence of pleomorphism and no other distinct cell types tend to be visualised.

- With regard to patterns of fibrosarcomas, it has been stated that the ensuing pattern types exist: [6]

$\circ \quad$ Keloid-like (thick hyalinised collagen fibres) pattern.

o Loose fascicular pattern.
○ Focally myxoid pattern.

\section{Staining Features \\ Positive Staining}

- The staining features of fibrosarcomas have been summated as follows: [6]

- Reticulin stain does demonstrate fibres encompassing each cell.

- Phosphotungstic acid-haematoxylin does demonstrate an abundance of cytoplasmic fibrils.

- Immunohistochemistry staining studies would show that the tumour cells stain positively with:

i. Vimentin.

ii. Type 1 collagen.

iii. P53

- Immunohistochemistry staining does show High Ki-67 staining activity.

- Immunohistochemistry staining may exhibit CD34 positive staining if the tumour does arise from DFSP or solitary fibrous tumour.

\section{Negative staining}

- It has been documented that immunohistochemistry staining studies in fibrosarcomas tend to show negative staining for [6]

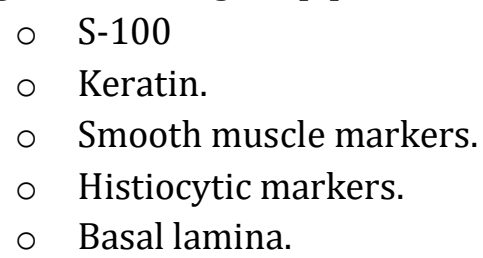

\section{Electron microscopy examination} features.

- The electron microscopy examination features of fibrosarcomas have been summated as follows: [6] 
$\circ$ Electron microscopy examination of fibrosarcomas tends to show fibroblasts that have prominent rough endoplasmic reticulum with no evidence of myofilaments and no evidence of external lamina as well as no evidence of intercellular junction.

○ With regard to fibrosarcoma, electron microscopy examination does not show any distinct myofibroblasts and if myofibroblasts are present then the tumour would be called myofibrosarcoma.

\section{Molecular and Cytogenetics Examination Features:}

- It has been iterated that molecular and cytogenetics studies of fibrosarcmas tend to show that the tumours exhibit Aneuploidy [6].

\section{Differential diagnosis}

- It has been iterated that the differential diagnoses of fibrosarcomas include other tumours that contain fibrosarcomatous parts including [6]

- Dedifferentiated liposarcomas

- Fibromatosis which tends to be less-cellular, to have less hyperchromasia, tends not to have any atypia, tends to have less than 1 mitotic figure per high power field.

○ Low-grade fibromyxoid sarcoma.

○ MFH-pleomorphic.

- MPNST

○ Synovial sarcoma.
(A) Miscellaneous narrations and discussions from some case report, case series, and studies related to primary angiosarcoma of the testis and intra-scrotal contents including the epididymis, tunica, spermatic cord, and other paratesticular tissues.

Taha et al. [7] reported a 21-year-old man who had presented with right intra-scrotal swelling which had increased in size insidiously over a period of three months. He was asymptomatic otherwise. He did not have any problem related to his left hemi-scrotal contents. His clinical examination revealed a non-tender right testicular swelling that measured approximately $10 \mathrm{~cm}$, that was oval in shape and which was hard inconsistency. The scrotal skin and the left testis were normal. He had an ultrasound scan of the scrotum which showed a heterogeneous mass in his right testis. The results of his serum Human chorionic gonadotrophin and alphafetoprotein were within a normal range. He had computed tomography scan of thorax, abdomen, and pelvis which were normal. He underwent trans-inguinal right radical orchidectomy. Pathology examination of the specimen showed features that were consistent with the diagnosis of primary angiosarcoma of the testis. Microscopic histopathology examination of the specimen showed a hyper-cellular sarcomatous tumour which had consisted of a long fascicle of fusiform spindled-shaped cells that had been arranged in a herringbone pattern. The tumours had spindled-shaped nuclei and the cells of the tumour had been separated by interwoven collagen fibres that had been arranged in a parallel manner. Microscopy examination of the specimen also showed evidence of mild anaplasia to moderate anaplasia within the tumour as well as 
mitotic activity that was scattered (See figures 1 to 5). Immunohistochemistry staining studies of the tumour showed negative staining for SMA, S-100, and Desmin. Immunohistochemistry staining also showed that Ki67 was positively stained in $10 \%$ of the tumour which had denoted a low proliferation index. One month pursuant to his operation, he developed a swelling in his right hemi-scrotum that was painless and which had increased with regard to size. He had an ultrasound of his inguinal region and scrotum which showed a right testicular tumour and enlargement of his right inguinal lymph node. He underwent trans-inguinal resection of the recurrent scrotal mass and histopathology examination of the specimen showed an inflammatory process which had been dominated by stitch granuloma. Taha et al. [7] iterated that Catton et al. [8] had stated that with regard to patients who had undergone wide local excision and/or radiotherapy for para-testicular sarcomas, 14\% local recurrence had been documented. Taha et al. [7] additionally stated that Catton et al. [8] had made the ensuing summations related to intra-scrotal sarcomas:

- Orchidectomy alone would not be adequate treatment for sarcomas of the testis and they would recommend adjuvant wide hemi-scrotal excision, which could include inguinal lymph nodes for those individuals who are managed by means of orchidectomy or local excision of the tumour.

- They had found the presence of microscopic disease pursuant to wide excision of the tumour in $27 \%$ of patients who had undergone apparently complete wide excision of their tumours.

Taha et al. [7] stated that with regard to their reported case, a right intra-scrotal swelling recurred within one month of right radical orchidectomy which made assume the recurrent lesion was a recurrent fibrosarcoma but histopathology examination of the specimen they had excised at reexploration showed that the recurrent lesion was an inflammatory lesion. Taha et al. [7] additionally reported that they had followedup their patient for 6 months without finding any evidence of local recurrence or distant metastasis. It would be argued that the case of Taha et al. [7] was reported with a short period of follow-up and no none would know the long-term outcome of the patient and considering that fibroosarcomas could be aggressive tumours, there is the possibility that there could be local recurrence and or distant metastasis in the future and for this reason clinicians should be encouraged to report cases of fibrosarcoma of the testis they encounter with long-term outcome so that the biological behaviour of this rare malignancy would be known.

Zukenberg and Young [9] reported in 1990 two cases of pure primary testicular sarcomas. They reported that one tumour was osteosarcoma which was diagnosed in a 30-year-old man and the second case was a fibrosarcoma of the testis in an 86-year-old man. Both patients were treated by means of trans-inguinal radical orchidectomy without any adjuvant therapy. Both patients were alive at the time of the report of the cases in 1990.

In 1999, Val-Bernal et al. [10]stated that sarcoma of the testis would appear to be a very rare stromal tumour that usually tended to be associated with an indolent course with the potential for the development of distant metastases. Val-Bernal et al. [10] reported a 71-year-old man who had a pure primary fibrosarcoma of the testis. They reported that by the time the tumour became apparent the patient had already developed metastatic 
disease and the course of the disease was fatal. They also stated that at the time of the report of their case only one case of fibrosarcoma of the testis had been reported earlier.

Klein et al. [11] reported a case in which an abdominal low-grade fibrosarcoma had developed 4 years pursuant to completion of a 3-year treatment regimen of intensive combination therapy for metastatic nonseminomatouserm cell tumour of the testis.

O'Brien et al. [12] reported a case of radiotherapy-induced neurogenic fibrosarcoma which had developed in a patient who had undergone radiotherapy for seminoma of the testis. The fibrosarcoma did develop within the radiotherapy field following a latency period of 19 years. O'Brien et al. [12] stated that even though the occurrence of a secondary malignancy tends to be unusual, the possibility of the development of fibrosarcoma should be included in the differential diagnosis of patients who do manifest with the growth of tumour after a long interval pursuant to having undergone radiotherapy.

McDonald. [13] reported a 16-year-old white boy, who in 1953 had presented with a painless left hemi-scrotal mass that had progressively enlarged over the preceding 2 months. He was asymptomatic otherwise. One month preceding his presentation he had had trauma to his scrotum. Upon examination, a hard tumour mass that measured $3 \mathrm{~cm}$ in diameter on the medial and superior part of his left testis was found. The left testis itself was normal. His examination was otherwise normal. The results of his urine examination, routine haematology blood test, and chest X-ray were normal. He underwent trans-inguinal left radical orchidectomy removing the entire tumour. Macroscopic examination of the specimen revealed that the testis was normal, the epididymis was normal, and arising from the tunica vaginalis a tumour mass that measured $4 \mathrm{~cm} \times 3 \mathrm{~cm} \times 3.5 \mathrm{~cm}$ was found which was hard, and irregular as well as glistening yellow-white in appearance. The tumour was completely separate from the spermatic cord, the epididymis, and the testis and upon sectioning the tumour was found to have whorled-features with almost looking like cartilage in some areas without any evidence of extension of the tumour into the adventitial tissues of the tunica vaginalis. The microscopy examination features of the tumour were consistent with the diagnosis of fibrosarcoma of the tunica vaginalis. The lesson to be learned from this case report is that even though fibrosarcoma of the tunica vaginalis testis is very rare, it should be considered in the differential diagnosis of tumours of the tunica vaginalis testis.

McCormack [14] in 1975 reported the $10^{\text {th }}$ case of primary fibrosarcoma of the epididymis. McCormack [14] stated that contrary to the majority of the previously reported cases, excision biopsy was ensued by a lengthy tumour-free period and a unique feature of the reported case was that of bilateral epididymal recurrence.

Blasco Alfonso et al. [15] reported a 55-yearold man who had para-testicular fibrosarcoma that presented in a most unusual way and which had developed very quickly. He had presented with an increase in the size of his left hemi-scrotum over a period of 6 months that had grown rapidly over the preceding 60 days. He was asymptomatic otherwise. His clinical examination revealed a left hemi-scrotal mass which did not transilluminate and which was dependent upon the spermatic cord but it was not adherent to the planes, and it b had measured $6 \mathrm{~cm}$ in diameter. There was no evidence of inguinal or supra-clavicular lymph node enlargement. 
The results of his serum beta-human chorionic gonadotrophin, alpha-fetoprotein, and lactate dehydrogenase were within a normal range. He had an ultrasound scan of the scrotum showed a heterogeneous solid mass that measured $5.4 \mathrm{~cm} \mathrm{x} 3.5 \mathrm{~cm}$ and which had exhibited hyper-echogenic signal with no apparent necrotic areas which had displaced the left testis medially (see figure 6). He had computed tomography (CT) scan of abdomen and pelvis which revealed a left hemi-scrotal mass which had displaced the left testis with regard to planes (see figure 7). There was no evidence of lymph node enlargement and no evidence of metastasis. He was treated by means of trans-inguinal radical orchidectomy without any adjuvant therapy. Macroscopic examination of the excised specimen revealed a $7 \mathrm{~cm}$ spermaticcord-dependent tumour. The tumour was a pseudo-encapsulated tumour. The paratesticular growth was found to be white in colour, multi-nodular, rubbery consistency and trabeculated with regard to texture as well as uniform to the section with no evidence of any area of necrosis or haemorrhage (see figure 8). Microscopic examination of the specimen revealed a homogeneous proliferation of fusiform cells within the mesenchymal tissue that had been arranged in confluent fascicles at an acute angle (see figure 9). The nuclei of the tumour cells were ovoid, regular, and 4 to 6 mitoses were seen per 10 high power fields. Gomori staining of the tumour showed that the tumour cells were encompassed by reticulin fibres (see figure 10). Immunohistochemistry staining studies of the tumour showed that the tumour cells had exhibited positive staining for vimentin, and negative staining for muscle markers, including smooth muscle action, and desmin. The KI-67 proliferation index was less than $1 \%$. The histopathology and immunohistochemistry staining features of the tumour were consistent with the diagnosis of low-grade para-testicular fibrosarcoma. Following 5 years of follow-up, there was no clinical or radiology imaging evidence of local recurrence or distant metastasis.

Martinez Izquierdo et al. [16] reported a 66year-old man who had undergone right orchidectomy in 2006 because of leiomyosarcoma of the epididymis. He was treated by means of, 50 Gy of adjuvant radiotherapy. He presented 5 years later with a left hemi-scrotal discomfort as well as a hard inguinal mass on the left side. He had an ultrasound scan of the scrotal contents and a computed tomography scan which revealed a left inguinal mass that measured $7 \mathrm{~cm} \mathrm{x} 6.8$ $\mathrm{cm}$ which was a suggestive recurrent tumour. He underwent radical left orchidectomy. Histology examination of the specimen showed neoplastic proliferation of elongated cells that had fusocellular appearance that was arranged in fascicles. The tumour cells had little cytoplasm and had infiltrated the adjacent fatty tissue. Microscopy examination also revealed atypia and pleomorphism with prominent hyperchromatic nuclei and nucleoli as well as intercellular collagen fibres. Immunohistochemistry staining studies of the tumour showed that the tumour cells had exhibited positive staining for vimentin and Bcl-2, focal positive staining for AE1/AE3, Desmin, and CD-10. Immunohistochemistry staining studies also showed that the tumour cells had exhibited negative staining for HMB-45, EMA, S-100, Calretinin, Caldesmon, CK5 / 7, total actin, smooth muscle actin, and smooth muscle myosin. The pathology examination features of the tumour were diagnosed as being consistent with the diagnosis of a $9 \mathrm{~cm}$ highgrade fibrosarcoma of the spermatic cord with the involvement of the surgical margin. The authors stated that the prognosis of fibrosarcomas tends to be poor with a 
survival of $50 \%$ due to the development of local recurrence or distant metastasis. They additionally stated the following:

- The treatment of choice for fibrosarcoma of the spermatic cord is radical orchidectomy with high ligation and section of the spermatic cord.

- Radiotherapy and/or adjuvant chemotherapy had not been adequately reported.

Ozbay et al. [17] reported a patient who had undergone left trans-inguinal radical orchidectomy for seminoma of the left testis and intraepithelial neoplasia of the right testis. Additionally, he received adjuvant radiotherapy of his para-aortic and left inguinal regions, and he also underwent local radiotherapy to his right testis. He developed fibrosarcoma of his left inguinal region and seminoma of his right testis 6 years pursuant to undergoing radiotherapy. He underwent wide excision of the left inguinal region fibrosarcoma and right partial orchidectomy. Two nodular tumour lesions developed within the lower pole of the right testis following his last intervention. He, therefore, underwent trans-inguinal right radical orchidectomy as well as hormone replacement therapy. A lesson that should be learned from this study is that radiotherapyinduced fibrosarcoma can subsequently develop in a previous radiation field following radiotherapy for seminoma of the testis as well as radiotherapy-induced seminoma of testis could subsequently develop following radiotherapy of the testis.

With regard to primary fibrosarcoma of the intra-scrotal contents on the whole very few cases have been reported with short followup data; hence the biological behaviour and long-term outcome is not known and therefore no global consensus opinion can be established regarding the best treatment option for the malignancy. The treatment options for the management of localized primary fibrosarcoma of the scrotal contents include: (a) radical complete excision of the lesion ensuring tumour-free surgical margin (trans-inguinal radical orchidectomy) alone, (b) radical orchidectomy plus chemotherapy, (c) radical orchidectomy plus radical adjuvant radiotherapy, (d) radical orchidectomy plus adjuvant chemotherapy plus adjuvant radiotherapy. With regard to locally advanced and metastatic fibrosarcoma of the scrotal contents, their treatment could include (a) radical orchidectomy plus retroperitoneal lymph node dissection alone, (b) radical orchidectomy plus retroperitoneal lymph node dissection plus radiotherapy, (c) radical orchidectomy plus retroperitoneal lymph node dissection plus chemotherapy, (d) radical orchidectomy plus retroperitoneal lymph node dissection plus chemotherapy plus radiotherapy. Despite treatment with the various available treatment options for apparently localized fibrosarcoma of the scrotal contents some patients subsequently develop local recurrence and others develop distant metastasis even though there was no evidence of distant metastasis or residual local disease at the time of the initial treatment. The 10-year survival rate of patients who undergo treatment for fibrosarcomas tend to be low and for this reason, it would be argued that perhaps radiotherapy and the available chemotherapy regimens are not very effective in the treatment of curative intent of the disease. The fact that recurrences could develop despite complete surgical excision of the tumour with complete tumour-free surgical margins and radiology imaging evidence of no residual tumour or distant metastasis would indicate that at the time of the initial surgical excision of the tumour there would have been 
microscopic metastatic tumours which were too small to be identified by the various radiology images and which subsequently grew bigger gradually to eventually become a clinical disease. Another issue that needs to be considered is the fact that radiotherapyinduced fibrosarcoma of the inguinoscrotal region has been documented pursuant to radical orchidectomy plus radiotherapy for seminoma of the testis. Considering the aforementioned factors it would be argued that perhaps radiotherapy would not be the best adjuvant treatment to be given in cases of fibrosarcoma of the scrotal contents and this will leave only chemotherapy as the only commonly available treatment option of curative intent. Considering that despite the use of chemotherapy in the treatment of fibrosarcomas, recurrences and progress of the disease does occur, it would be argued that perhaps some of the available chemotherapy medications are not effective and therefore there is a great need for urologists, oncologists, and researchers to undertake research in order to identify new chemotherapy options that would be more effective in destroying fibrosarcoma tumour cells. It could furthermore be argued that there is the need to undertake pre-operative

\section{Figure1:}

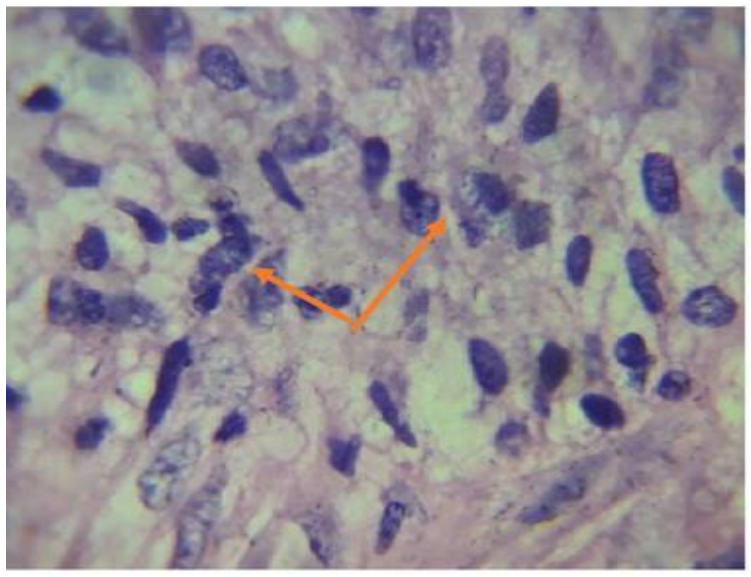

(A) radiology imaging-guided biopsies of all suspected scrotal content tumours for histopathology examination and if a diagnosis of primary fibrosarcoma is made the patients should be offered pre-operative neoadjuvant chemotherapy with the hope of destroying all of the possible microscopic metastatic tumours followed by radical orchidectomy (excision of tumour) and which should be followed by adjuvant combination chemotherapy with the hope of destroying any residual microscopic metastasis in order to avoid the development of subsequent clinical disease in the future. Additionally, it would be argued that urologists, researchers, and oncologists should explore the use of immunotherapy in the treatment of primary fibrosarcoma of the scrotal contents with long-term follow-up data. Finally, there the need for a global multi-center trial to be established to explore all the various treatment options for primary fibrosarcoma of the scrotal contents to identify the best treatment options for the management of the disease that would improve upon the longterm survival of the patients and also which would be associated with minimal side effects.

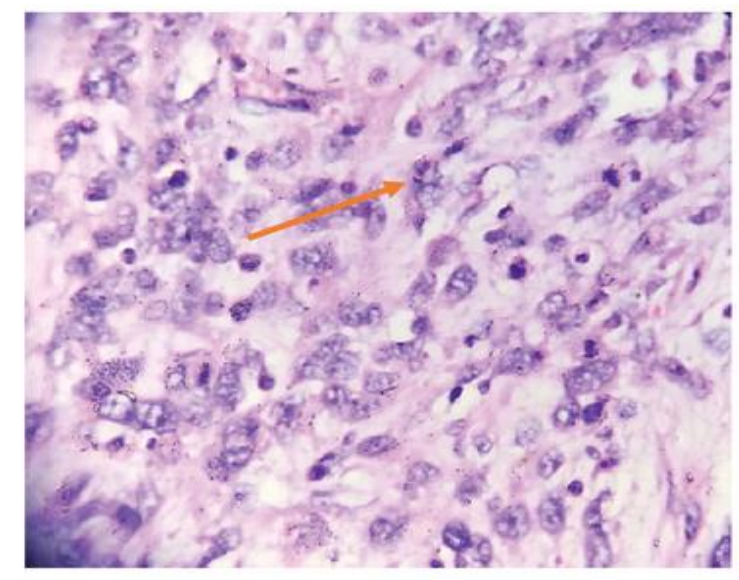

(B) 
Figure 1: Malignant hyper-cellular sarcomatous tumour formed with a long fascicle of fusiform spindle-shaped cells arranged in herringbone pattern spindleshaped nuclei with scanty cytoplasm and indistinct borders (orange arrows) Haematoxylin-eosin stain; magnification x 40 (A), magnification $x 100$ (B). The copyright of the image is kept for the authors (original authors). Reproduced from: [7] Taha S M, Osman Y M, Abuldris D, Ahmed M E I M, Awadalla M D, Mokhtar N M. Primary Fibrosarcoma of the Testicle with Puzzling

\section{Figure 2:}

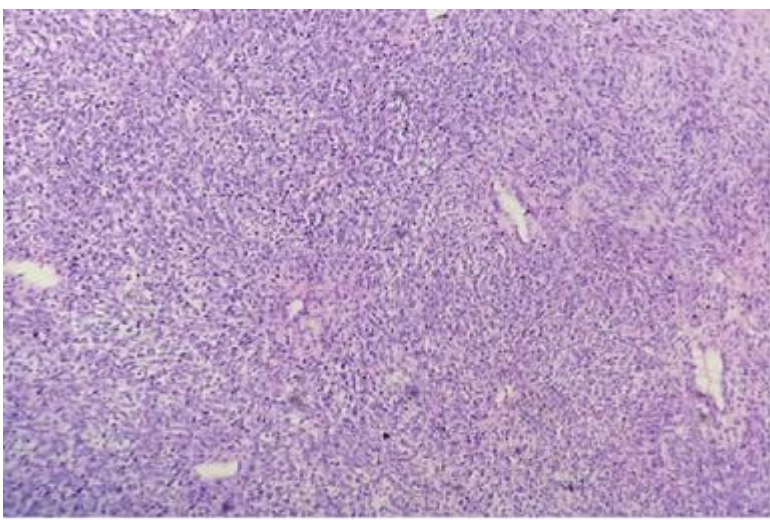

Figure 2: Collagen fibres arranged in parallel fashion: Haematoxylin-eosin stain; magnification x 10 (left) and x 20 (right). Reproduced from: [7] Taha S M, Osman Y M, Abuldris D, Ahmed M E I M, Awadalla M D, Mokhtar N M. Primary Fibrosarcoma of the Testicle with Puzzling Post-surgery Presentation: Mimicking Recurrence. KnE Publishing. Sudan Journal of Medical Sciences Figure 3:
Post-surgery Presentation: Mimicking Recurrence. KnE Publishing. Sudan Journal of Medical Sciences 2018; 13(2): DOI: 10.18502/sjms.v1312.2638

https://knepublishing.com/index.php/SJMS/ article/view/2638/5655\#figures Under copyright \& license: copyright (C) 2018 Sami Taha et al. [7] This article is distributed under the terms of the Creative Commons Attribution License, which permits unrestricted use and redistribution provided the original author and source are credited.

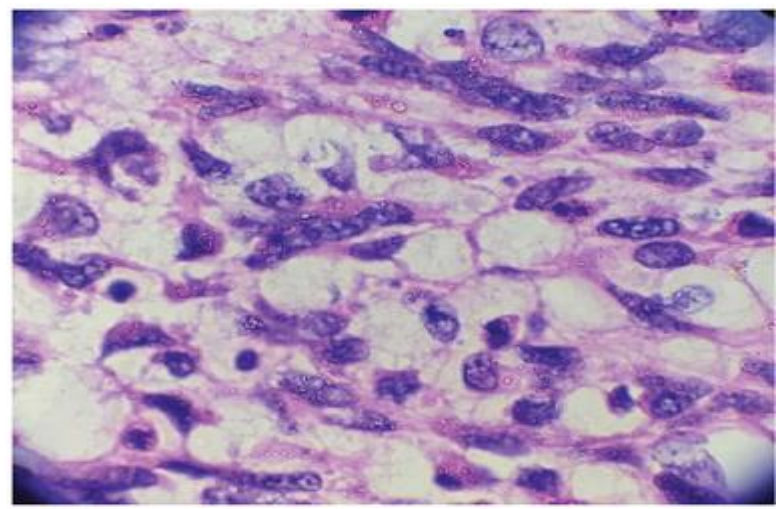

2018; 13(2): DOI: 10.18502/sjms.v1312.2638 https://knepublishing.com/index.php/SJMS/ article/view/2638/5655\#figures Under copyright \& license: copyright (C) 2018 Sami Taha et al. [7] This article is distributed under the terms of the Creative Commons Attribution License, which permits unrestricted use and redistribution provided the original author and source are credited.

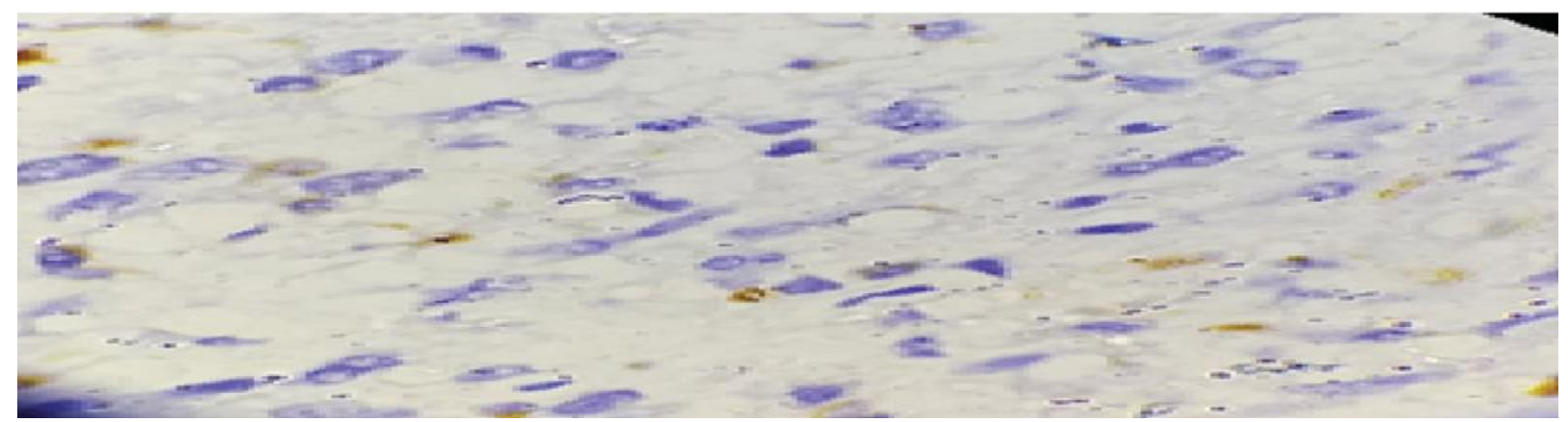

International Research in Medical and Health Sciences | Vol. 2 | Issue 4 | July- August| 2019

Page 17 
Figure 3: Ki67 immunochemistry is positive - in about $10 \%$ of tumour cells (magnification x 40). Reproduced from: [7] Taha S M, Osman Y M, Abuldris D, Ahmed M E I M, Awadalla M D, Mokhtar N M. Primary Fibrosarcoma of the Testicle with Puzzling Post-surgery Presentation: Mimicking Recurrence. KnE Publishing. Sudan Journal of Medical Sciences 2018; 13(2): DOI: 10.18502/sjms.v1312.2638

\section{Figure 4:}

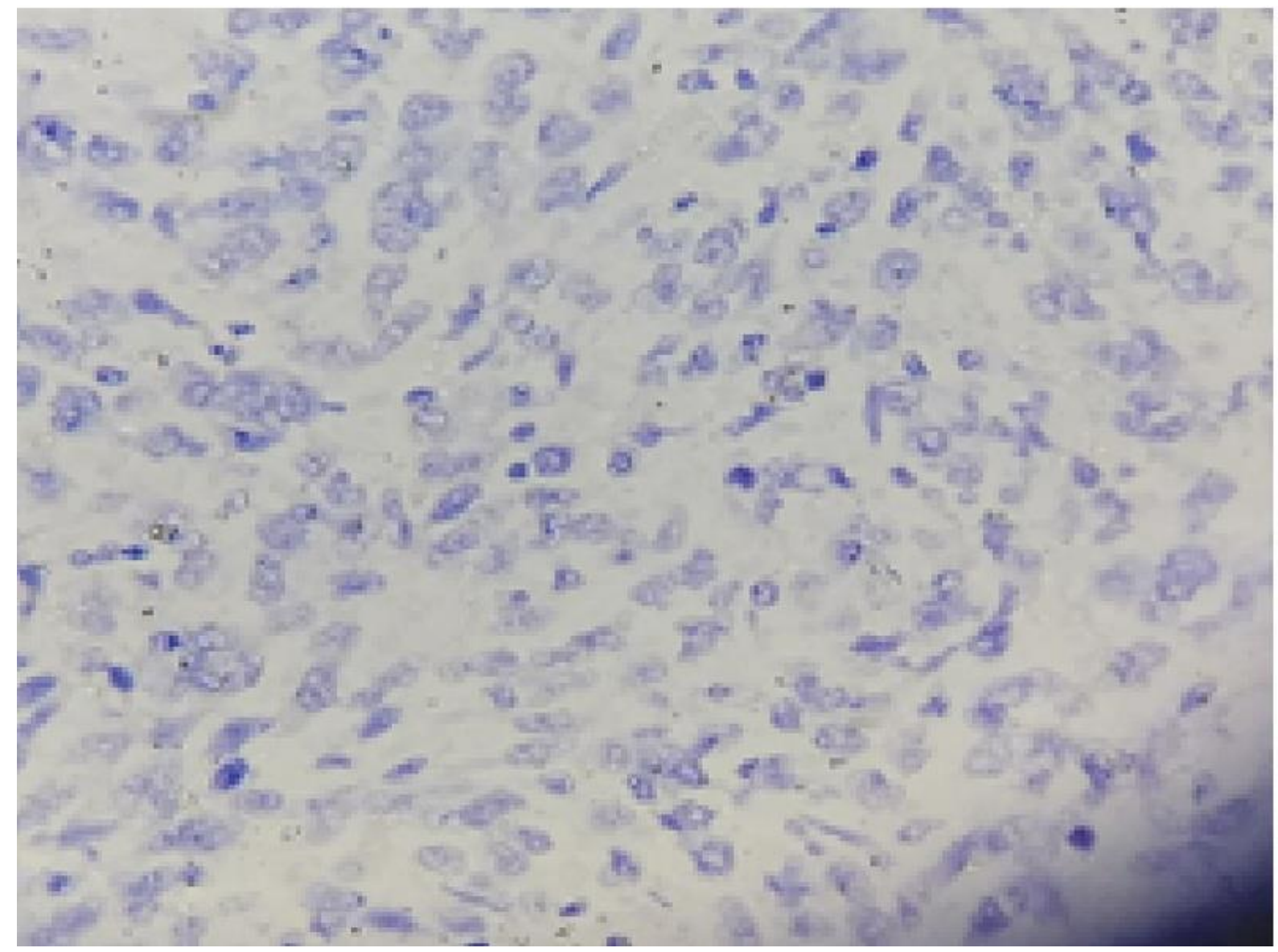

Figure 4: $\mathrm{S} 100$ is negative - immunochemistry (magnification $x \quad 40$ ). Reproduced from: [7] Taha S M, Osman Y M, Abuldris D, Ahmed M E I M, Awadalla M D, Mokhtar N M. Primary Fibrosarcoma of the Testicle with Puzzling Post-surgery Presentation: Mimicking Recurrence. KnE Publishing. Sudan Journal of Medical Sciences 2018; 13(2): DOI: 10.18502/sjms.v1312.2638
https://knepublishing.com/index.php/SJMS/ article/view/2638/5655\#figures Under copyright \& license: copyright (c) 2018 Sami Taha et al. [7] This article is distributed under the terms of the Creative Commons Attribution License, which permits unrestricted use and redistribution provided the original author and source are credited. 


\section{Figure 5:}

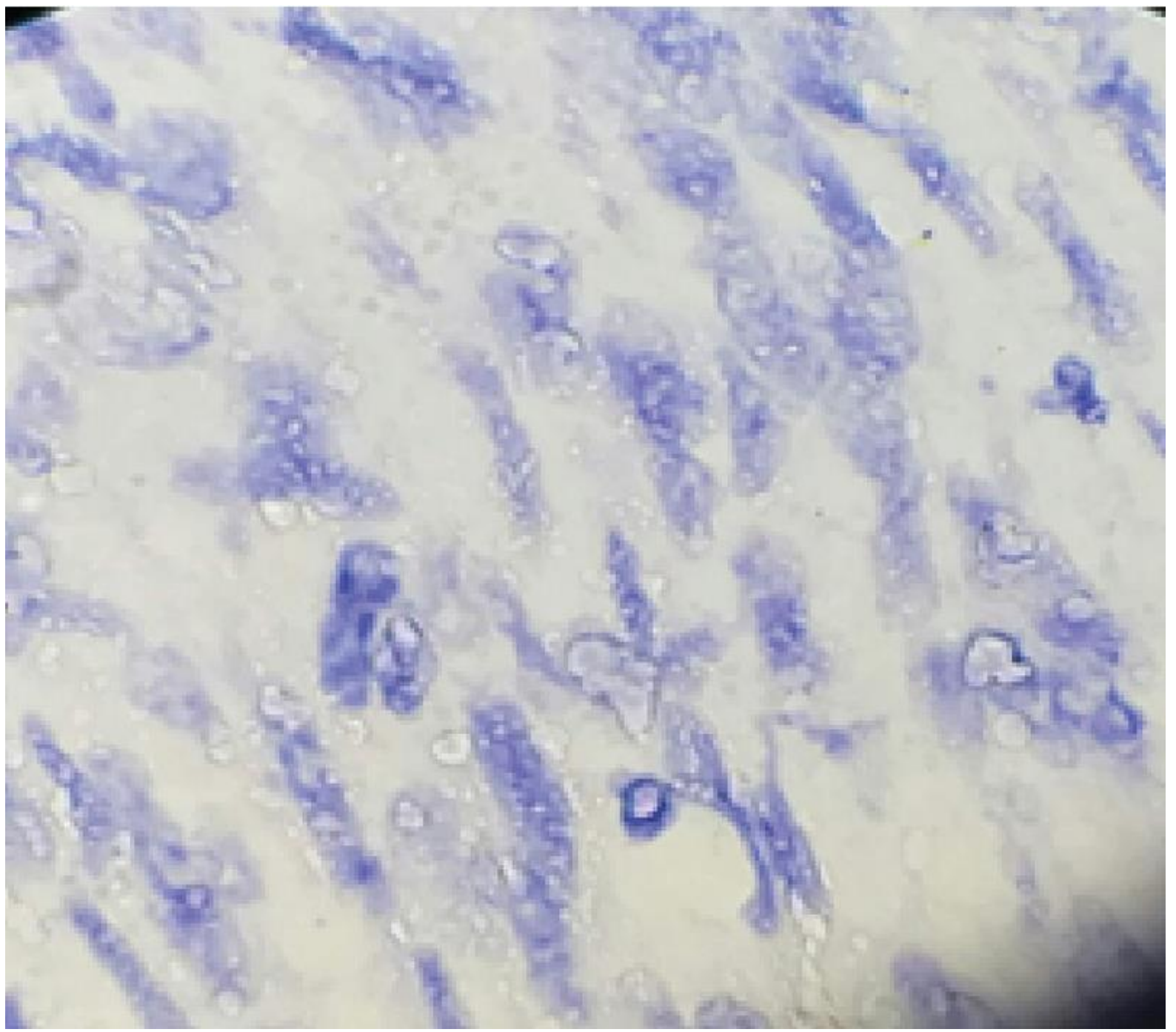

Figure 5: Desmin is negative - immunochemistry (magnification $x$ 40). Reproduced from: [7] Taha S M, Osman Y M, Abuldris D, Ahmed M E I M, Awadalla M D, Mokhtar N M. Primary Fibrosarcoma of the Testicle with Puzzling Post-surgery Presentation: Mimicking Recurrence. KnE Publishing. Sudan Journal of Medical Sciences 2018; 13(2): DOI: 10.18502/sjms.v1312.2638 https://knepublishing.com/index.php/SJMS/

\section{article/view/2638/5655\#figuresUnder} copyright \& license: copyright (c) $2018 \mathrm{Sami}$ Taha et al. [7] This article is distributed under the terms of the Creative Commons Attribution License, which permits unrestricted use and redistribution provided the original author and source are credited. 


\section{Figure 6:}

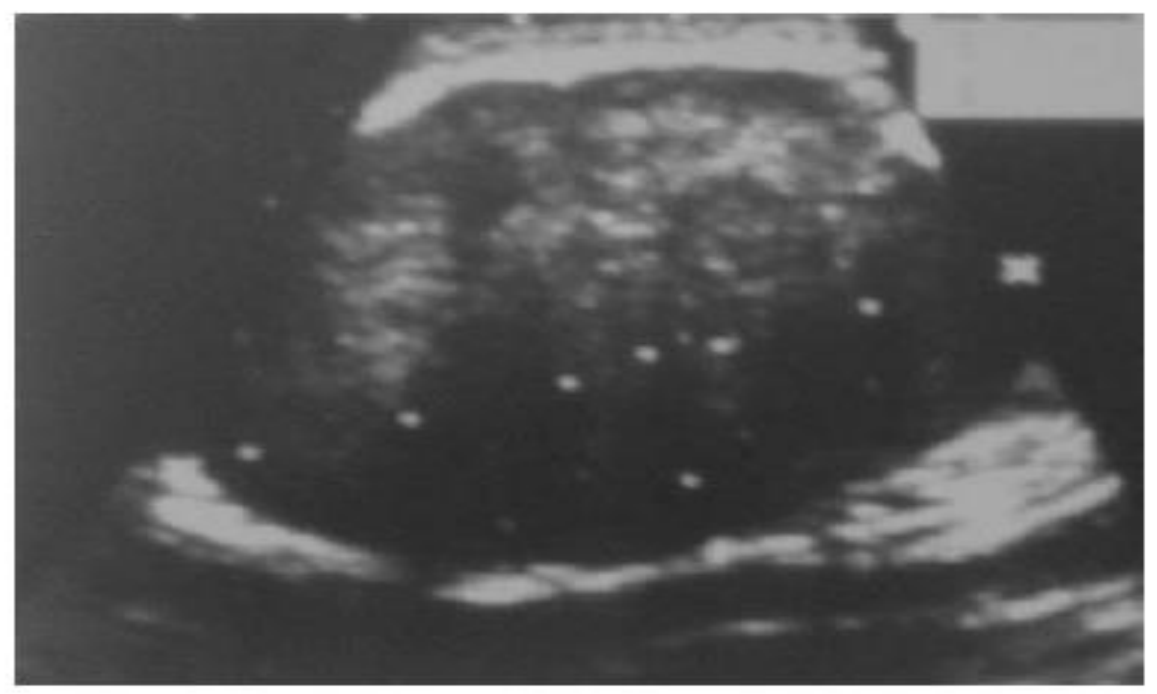

Figure [6] Ultrasound scan shows a heterogeneous hyper-echogenic solid mass

Reproduced from: [15] Blasco Alfonso J E, Sales Maicas M A. Fibrosarcomaparatesticular: una neoplasia malignamuyinfrecuente. (Paratesticularfibrosarcoma: A rare malignant neoplasm). Actas Urol. Esp. 2006 Jul-Aug; 30(7): 707710 DOI: https://doi.org/10.1016/S02104806(06)73521-5http://scielo.isciii.es/scielo.php?script=sci arttext\&pid=S0210-

48062006000700009 under copyright: all the contents of this magazine, except where it is identified, is under the Creative Commons Attribution License CC BY-NC.

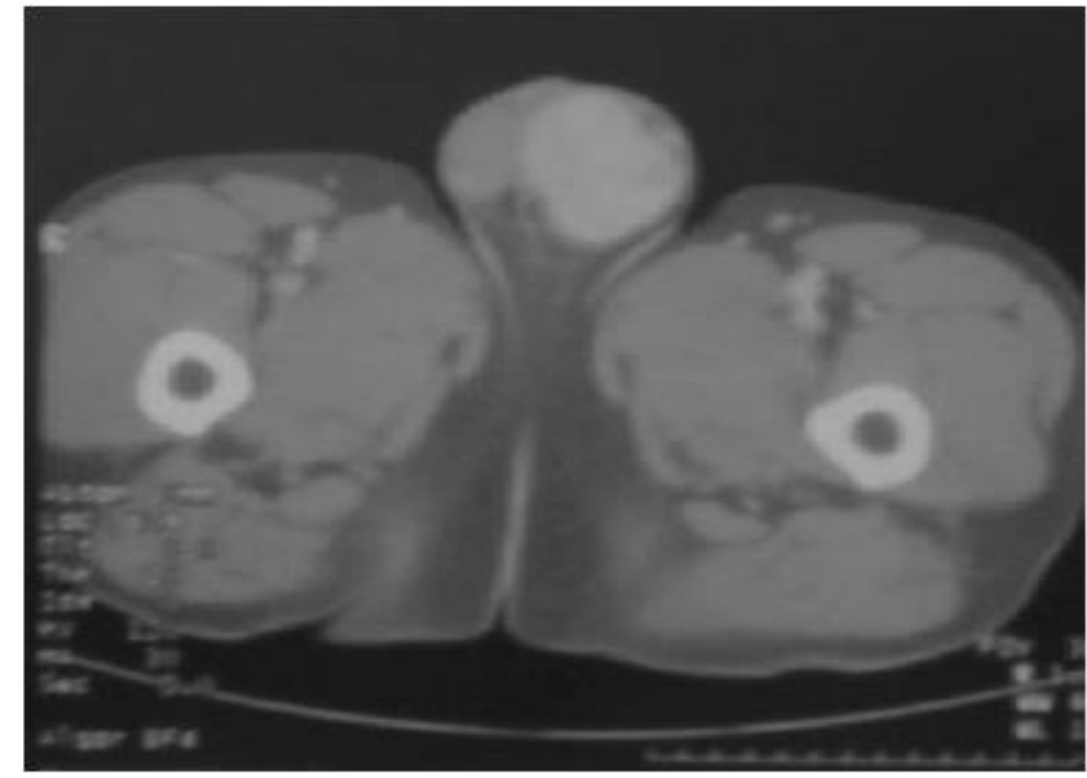

Figure 7: Computed tomography scan of the abdomen and pelvis left hemi-scrotal mass which had displaced the left testis. Reproduced from: [15] Blasco Alfonso J E, Sales Maicas M A. 
Fibrosarcomaparatesticular: una neoplasia malignamuyinfrecuente. (Paratesticularfibrosarcoma: A rare malignant neoplasm). Actas Urol. Esp. 2006 Jul-Aug; 30(7): 707710 DOI: https://doi.org/10.1016/S0210-4806(06)73521-

5http://scielo.isciii.es/scielo.php?script=sci arttext\&pid=S0210-48062006000700009 under copyright: all the contents of this magazine, except where it is identified, is under the Creative Commons Attribution License CC BY-NC.

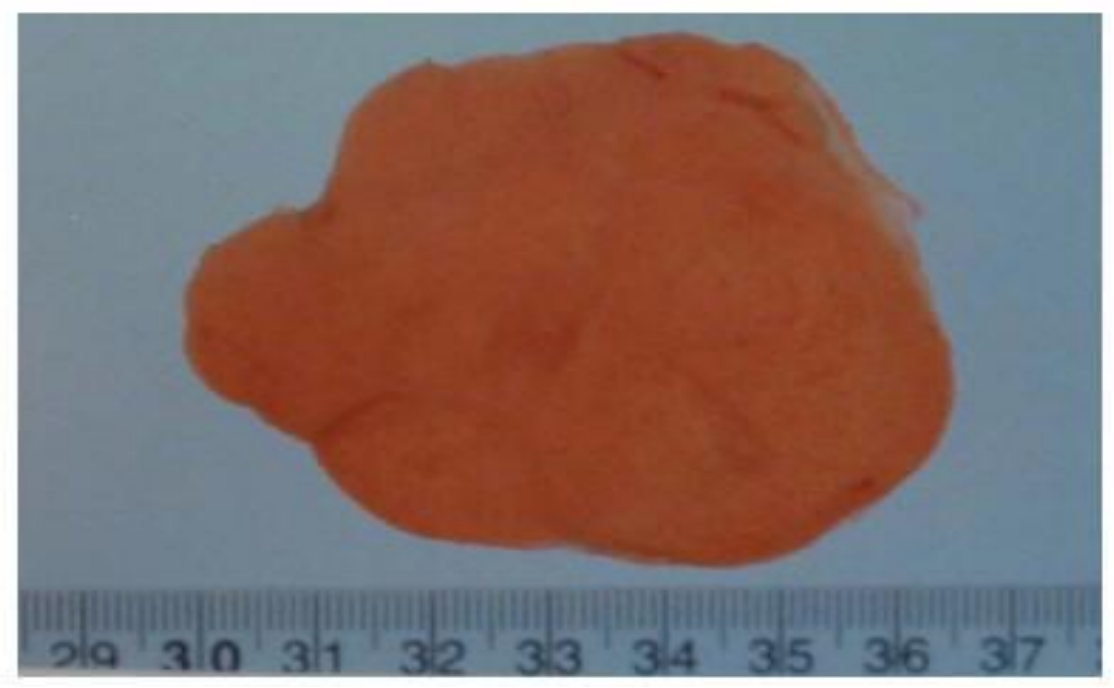

Figure 8: A white tumour, pseudo-encapsulated, uniform, with no areas of haemorrhage or necrosis. Reproduced from: [15] Blasco Alfonso J E, Sales Maicas M A. Fibrosarcomaparatesticular: una neoplasia malignamuyinfrecuente. (Paratesticularfibrosarcoma: A rare malignant neoplasm). Actas Urol. Esp. 2006 Jul-Aug; 30(7): 707710 DOI: https://doi.org/10.1016/S02104806(06)73521-5http://scielo.isciii.es/scielo.php?script=sci arttext\&pid=S0210-

48062006000700009 under copyright: all the contents of this magazine, except where it is identified, is under the Creative Commons Attribution License CC BY-NC.

Figure 9:

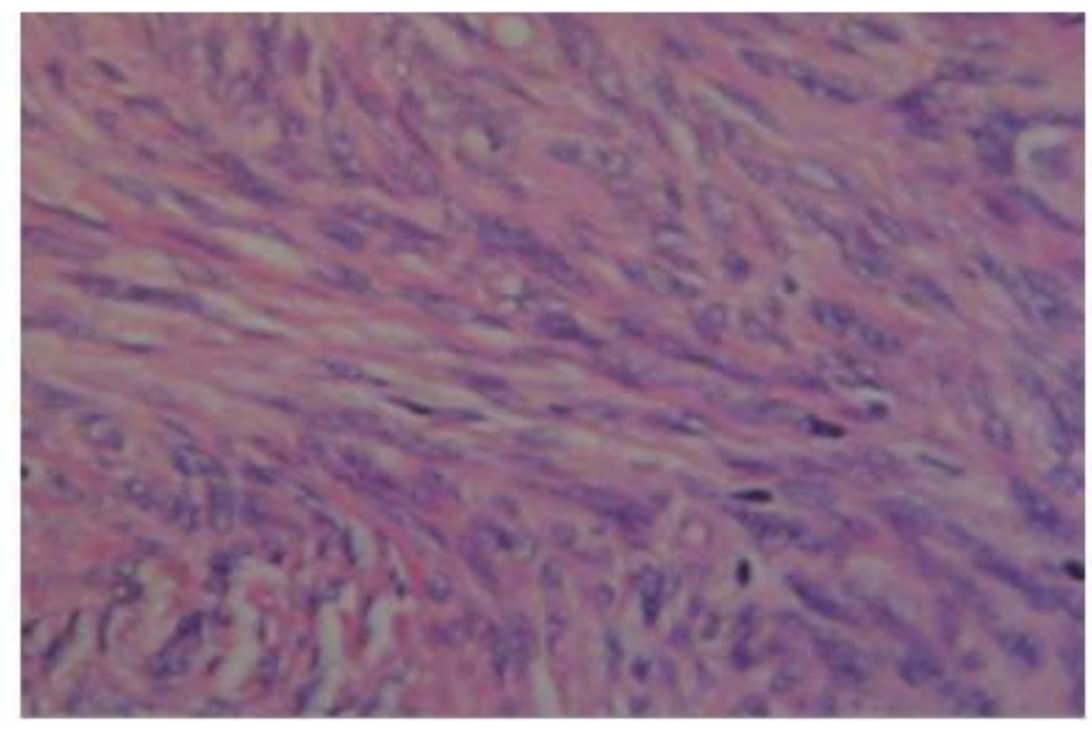


Figure 9: Haematoxylin \& Eosin $x$ 40. Homogeneous proliferation of fusiform cells within the mesenchymal tissue Reproduced from [15] Blasco Alfonso J E, Sales Maicas M A. Fibrosarcomaparatesticular: una neoplasia malignamuyinfrecuente. (Paratesticularfibrosarcoma: A rare malignant neoplasm). Actas Urol. Esp. 2006 Jul-Aug; 30(7): 707710 DOI: https://doi.org/10.1016/S0210-4806(06)73521-

5http://scielo.isciii.es/scielo.php?script=sci arttext\&pid=S0210-48062006000700009 under copyright: all the contents of this magazine, except where it is identified, is under the Creative Commons Attribution License CC BY-NC.

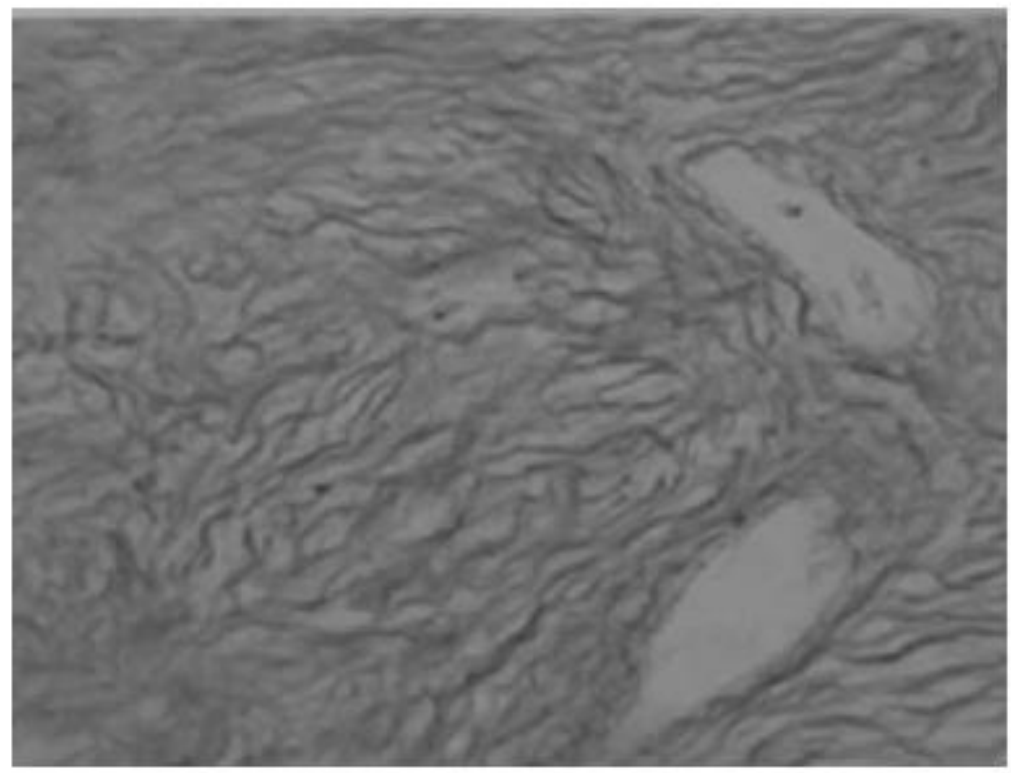

Figure 10: Gomori staining of the tumour magnification 40x; showing that the tumour cells are surrounded by reticulin fibres. Reproduced from: [15] Blasco Alfonso J E, Sales Maicas M A. Fibrosarcomaparatesticular: una neoplasia malignamuyinfrecuente. (Paratesticularfibrosarcoma: A rare malignant neoplasm). Actas Urol. Esp. 2006 Jul-Aug; 30(7): 707710 DOI: https://doi.org/10.1016/S0210-4806(06)73521-

5http://scielo.isciii.es/scielo.php?script=sci arttext\&pid=S0210-48062006000700009 under copyright: all the contents of this magazine, except where it is identified, is under the Creative Commons Attribution License CC BY-NC.

\section{Conclusions and Recommendations:}

- Primary fibrosarcoma of the intrascrotal contents is a very rare malignancy which has been treated in sporadically by various options including radical orchidectomy alone, radical orchidectomy plus adjuvant therapy including radiotherapy plus / minus / or chemotherapy with on the whole a short-term and medium-term follow-up.

- On the whole, the long-term prognosis of primary fibrosarcomas has been low.

- The subsequent development of radiotherapy-induced fibrosarcoma in the inguinoscrotal region following radical orchidectomy plus radiotherapy for seminoma of the testis has been reported which would 
suggest that perhaps adjuvant radiotherapy for testicular malignancies may not be an ideal treatment.

- Considering that the 10-year survival of patients who have undergone various treatments for fibrosarcoma, on the whole, is low, there is the need to identify through careful research new chemotherapy combination chemotherapy medicaments that would effectively destroy fibrosarcoma tumour cells at the onset of treatment to avoid the subsequent development of local recurrence and distant metastasis after radical orchidectomy for localized fibrosarcoma of the scrotal contents. This could possibly be achieved through a long-term global multi-centre clinical trial.

- All patients who have intra-scrotal masses that are clinically suspicious to be malignant tumours should be encouraged to undergo pre-operative radiology image-guided biopsy of the lesion for histopathology examination and if a diagnosis of fibrosarcoma is made they should be considered for neoadjuvant chemotherapy if the tumour is large, poorly differentiated or does contain a high mitotic index and this should be followed by radical orchidectomy with tumour-free surgical margin and adjuvant chemotherapy.

- Urologists and oncologists should consider including immunotherapy in the treatment for primary fibrosarcoma of the scrotal content as additional treatment after they had undertaken a global study to confirm this mode of treatment would be effective.

\section{Conflict of interest - None Acknowledgments to:}

Actas Urol. Esp. for granting permission for reproduction of figures and contents of their journal article under copyright: all the contents of this magazine, except where it is identified, is under the Creative Commons Attribution License CC BY-NC.

KnE Publishing and Sudan Journal of Medical Sciences for granting permission for reproduction of figures and contents of their journal article under copyright \& license: copyright (C) 2018 Sami Taha et al., [7] This article is distributed under the terms of the Creative Commons Attribution License, which permits unrestricted use and redistribution provided the original author and source are credited.

\section{References}

1. Dickey I D. Floyd J G. Chansky H A, Chief Editor Binitie O. Fibrosarcoma Medscape Updated 2018 Dec 03

2. Antonescu C R, Erlandson R A, Huvos A G. Primary fibrosarcoma and malignant fibrous histiocytoma of bone- a comparative ultrastructural study: evidence of a spectrum of fibroblastic differentiation. Ultrastructpathol. 2000; Mar-Apr; 24(2): 83 - 89]

3. Lin C N, Chou S C, Li C F, Tsai KB, Chen W C, Hsiung C Y, Yen CF, Huang H Y. Prognostic factors of myxofibrosarcomas: implications of margin stats, tumour necrosis, and mitotic rate on survival. J Surg Oncol. 2006; 93(4): $294-303$

4. Nakanishi H, Tomita $\mathrm{Y}$, Ohsawa $\mathrm{M}$, Naka N, Araki N, Ochi T, Aozasa K. 
Tumor size as a prognostic indicator of histologic grade of soft tissue sarcoma. J. Surg Oncol. 1997 Jul. 65(3): 183 - 187.

5. Augsburger D, \nelson P J, Kalinski T, Udelnow A, Knosel T, Hofstetter M, Qin J W, Wang Y, Gupta A S, Bonifatius S, Li M, Bruns C J, Zhao Y. Current diagnostics and treatment of fibrosarcoma - perspectives for future therapeutic targets and strategies. Oncotarget. 2017 Nov 28; 8(61); 104638 - 104653. DOI: 10.18632/oncotarget.20136

6. Arora K. Soft tissue fibroblastic / myofibroblastictumorsFibrosarcoma of soft tissue - adult.

PathologyOutlines.com Topic Completed 2012 Jul 01; Revised 2019 Mar 21.

http://www.pathologyoutlines.com/t opic/softtissuefibrosarcoma.html. Accessed 2019 Aug 16

7. Taha S M, Osman Y M, Abuldris D, Ahmed M E I M, Awadalla M D, Mokhtar N M. Primary Fibrosarcoma of the Testicle with Puzzling Postsurgery Presentation: Mimicking Recurrence. KnE Publishing. Sudan Journal of Medical Sciences 2018; 13(2):

DOI: 10.18502/sjms.v1312.2638

8. Catton C N, Jewett M, O'Sullivan B, Kandel R. The Journal of Urology 1999; 161: 1844-1847 DOI: http://dx.doi.org/10.1016/S002253470(05068823-4

9. Zukenberg L R, Young R H. primary testicular sarcoma: A report of two cases. Human Pathology 1990; Sep; 21(9): 932 - 935. https://doi.org/10.1016/00468177(90)90177-7

10. Val-Bernal J F, Azcarretazábal T, Torío B, Mayorga M. Primary pure intratesticularfibrosarcoma. PatholInt 1999 Feb; 49(2): 185 -189

11. Klein F A, Herr H W, Vugrin D. Fibrosarcoma associated with intensive chemotherapy for advanced germ cell testicular tumor. Journal of Surgical Oncology 1983 May; 23(1): https://doi.org/10.1002/jso.2930230 103.

12. Obrien W M, Abbondanzo S L, Chun B, Manz H J, Maxted W C.Neurogenicfibrosarcoma following radiation therapy for seminoma. Urology 1989; 33(5): 420 - 423

13. McDonald J H. Primary fibrosarcoma of the tunica vaginalis. The journal of Urology 1955; 73(6): 1069 - 1074.

14. McCormack M M. Bilateral fibrosarcoma of the epididymis. Journal of clinical pathology 1975; 28(7): 576 - 579

15. Blasco Alfonso J E, Sales Maicas M A. Fibrosarcomaparatesticular: una neoplasia malignamuyinfrecuente. (Paratesticularfibrosarcoma: A rare malign neoplasm). Actas Urol. Esp. 2006 Jul-Aug; 30(7): 707710 DOI: https://doi.org/10.1016/S02104806(06)73521-5

16. Martinez Izquierdo $\mathrm{M} A$, Blasco Martinez A, Cabrejas Morales R, Lopez Garcia-Asenjo J A.Spermatic Cord Fibrosarcoma SEAP-IAP Annual Meetings XXXV SEAP Annual Meeting 2012 Feb 16 and 17; Hotel Rafael Atocha C / MAENDEZ Alvaro 30-32 28045 Madrid Posters https://seap.es 
17. Ozbay B, Kernahli E, Tugcu V, Tasci A I. A case of radiation-induced fibrosarcoma following radiotherapy for testicular seminoma BTDMJB 2007; $3(2): 81 \quad-\quad 83$. https://www.scopemed.org/?mno=4 $\underline{0179}$ Accessed 2019 Aug 17

How to cite this Article: Anthony Kodzo-Grey Venyo; Primary Fibrosarcoma of the Testis and Other Scrotal Contents: A Review and Update Int. Res. Med. Health Sci., 2019; 2(4):1-25.

Source of Support: Nil, Conflict of Interest: None declared.

Received: 25-07-2019; Revision: 14-08-2019; Accepted: $30-08-2019$ 